\title{
Dynamic Scheduling of Virtual Cellular Based on Rolling Window under New Tasks
}

\author{
Wenmin Han, Yi Yu, Ting Chen and Zhengyi Li \\ School of Economics and Management, Jiangsu University of Science and Technology, Jiangsu \\ Zhenjiang 212003, China
}

Keywords: dynamic scheduling of virtual cellular, rolling window, multi-objective genetic algorithm

\begin{abstract}
Aiming at the dynamic scheduling problem of virtual cellular generated by the random arrival of new tasks, combined with the rolling window technology, the decision-making judgment based on the order completion trigger and the machine idle state trigger is put forward. At the same time, the dynamic random scheduling period is divided into continuous interval of static scheduling. And a non-linear multi-objective 0-1 integer programming model is proposed, which is based on the maximum completion time, the weighted total delay and the initial scheduling degree of deviation as the targets. The multi-objective genetic algorithm is used to solve the model. Finally, taking the shipbuilding as an example, the feasibility and effectiveness of the rescheduling model are verified.
\end{abstract}

\section{Introduction}

The virtual cellular mainly classifies the workpiece into the product family according to the proces similarity and distributes resources similarity of the production logically to the corresponding family of products. When the processing task is finished, the equipment in the virtual cellular is automatically released for other virtual cellular to use, with the advantage of improving the flexibility and agility [1]. The random arrival of the new tasks is one of the most common perturbation factors in the actual production process. As a result of the random arrival of the new task, the virtual cellular need to carry on the dynamic rescheduling.

From the existing results, the research on the problem of rescheduling at home and abroad mainly focuses on the optimization of the rescheduling method and the evaluation of the performance of the rescheduling [2-3]. The study of rescheduling can be divided into three types: cycle-driven, event-driven and mixed-driven scheduling. Adibi [4] study the dynamic scheduling problem of Job Shop manufacturing system and select the arrival of new workpiece and machine failure as a decision point of rescheduling. Zhang Jie [5] designed the rolling scheduling strategy based on the tolerance of the delivery time deviation for the dynamic scheduling of the mixed flow shop. Yin [6] studied dynamic scheduling under disruption of machines and constructed a multi-objects model which considers both the balance of system and the minimum of makespan. Qiao [7] considered the uncertain of semiconductor productive lines, established a model of re-scheduling fuzzy PETRI network. Liu Ming Zhou [8] constructed the rescheduling profit and loss function of the manufacturing shop, and suggested whether the rescheduling was needed, from the view of the cost of rescheduling execution.

In order to balance the stability and agility of virtual cellular manufacturing system better, this paper proposed a new type of redistribution driving decision, which aims at dynamic reconfiguration problem of the virtual cellular based on the random arrival of new tasks. Combined with the rolling window rescheduling technique, this paper proposed a nonlinear multi-objective $0-1$ integer programming model, which considered the maximum completion time, the weighted total delay and the initial scheduling deviation. Meanwhile, an optimized multi-objective genetic algorithm is designed. 


\section{Rescheduling Driven Decision}

\subsection{Rolling Window Technology.}

The main idea of the rolling window technology for dynamic scheduling is to roll for optimization. When the new orders arrive, the actual scheduling and pre-scheduling program will produce bias, and the completed workpiece from the window need to be moved. The part of the workpiece (including the new tasks) will be selected to join the rolling window until all orders have been completed. In order to handle the new tasks well, the rolling window based on the process is adopted.

\subsection{The Rescheduling Driven Decision of New Tasks.}

The new task belongs to the emergency task and the delivery time is short, if the rescheduling is not carried out, which will cause great loss to the company. At the same time, the machine load of the virtual cellular production system is not balanced, and the average load is low. Therefore, this paper presents a new type of driven method in the mode of hybrid driven. The trigger of the rescheduling is the completion time and the idle state of machine.

\subsubsection{Trigger of task Completion time}

The buffer time can be expressed as:

$$
\bar{T}=\max \left(0, d_{i}-t-\sum_{j=1}^{J_{i}} N_{i} T_{i j}\right)
$$

Where the $\bar{T}$ indicates that the time can be buffered, $d_{j}$ represents the delivery time of the workpiece, $t$ represents the current dispatching time, $N_{i}$ represents the number of workpieces, $T_{j i}$ represents the processing time for the step $j$ of the workpiece $i, J_{i}$ represents the number of steps for the workpiece $i$, the threshold is set to be greater than 0 .

\subsubsection{Trigger of machine idle warning}

Expected using rate of machine can be expressed as:

$\bar{U}_{\mathrm{m}}=\frac{\sum_{m=1}^{M} \frac{T_{\mathrm{m}}}{T_{m}^{\max }-\mathrm{t}}}{M}$

Where $\bar{U}_{\mathrm{m}}$ represents the average utilization rate of the machine. $M$ represents the total number of machines, $t$ represents the current dispatching time, $T_{m}$ represents the processing time of the machine in the pre-scheduling scheme. $T_{m}^{\max }$ represents the maximum processing time in all machines in the pre-scheduling program. When the threshold $\bar{U}_{\mathrm{m}}$ is exceeded, the restart is initiated. In this paper, threshold $\bar{U}_{\mathrm{m}}$ is setted to be more than 0.3 .

\section{Rescheduling Model}

\subsection{The Description of the Problem and Assumptions.}

In the hybrid driven mechanism, any trigger triggered must be rescheduled. Therefore, the delivery time and the maximum completion time should be considered. At the same time, scheduling stability is also an important index needrd to be considered [9-11]. In order to make the research more feasible, this study is based on the following assumptions:

(1)The same work piece can only be processed by one machine at the same time; (2) Any process will not be interrupted once the equipment is started; (3) The state of each device in the current rescheduling time is determined by the actual scheduling scheme of the previous scheduling interval; (4) The new tasks can be scheduled in the next scheduling interval in the scheduling interval; (5) Adjustment time of the equipment is included in the processing time.

Define variables in optimization model as follows: $B T_{i_{i} j m}$ represents the start time of the machining workpiece $i$ on equipment $m$ in procedure $j$ under rout $r_{i} ; B T_{j}$ represents the time when procedure $j$ initially schedules procedure to start machining; $B T_{j}^{*}$ represents the procedure $j$ newly schedules procedure to start machining; $y_{m}$ represents the total procedure yield increased or reduced from initial 
schedule; $E T_{i_{r} j m}$ represents the terminal time of machining workpiece $i$ on equipment $m$ in procedure $j$ under rout $r_{i} ; P_{i_{i} j m}$ represents the time of machining workpiece $i$ on equipment $m$ in procedure $j$ under rout $r_{i} ; T P_{i}$ represents the lead time of workpiece $i ; D_{r_{i}}$ represents the machining batch in rout $r_{i} ; S_{i}$ represents the arrival time of workpiece $i ; t_{0}$ represents the start time of rescheduling in current scheduling interval; $T_{k}(k=1, \cdots, K)$ represents the No. k rescheduling time; $J_{k}$ represents the mark number set of workpiece within No. $k$ rescheduling interval, $J$ can stand for a given parameter, where $J_{0}$ stands for the mark number set of workpiece within No. 1 scheduling interval, time quantum belongs to $t_{0} \sim T_{1} ; A C_{m}$ represents the maximum unit throughput of equipment $m$ within planned interval; $H$ represents the length of planned interval.

\subsection{Optimization Model and Constraints.}

This paper builds a dynamic virtual cellular nonlinear integer scheduling model considering the minimum makespan and total weighted tardiness, as follows:

$$
\begin{gathered}
F=\min \left\{f_{1}, f_{2}, f_{3}\right\} \\
f_{1}=\max _{i=1}^{I} \max _{r_{i}=1}^{R_{i}}\left(B T_{i r_{i} J_{i}}+D_{r_{i}} P_{i_{i} J_{i} m}\right) \\
f_{2}=\sum_{i=1}^{I} \sum_{r_{i}=1}^{R_{i}}\left\{\max \left[\omega_{i}\left(E T_{i_{i} J_{i}}-T P_{i}\right), 0\right]\right\} \\
f_{3}=\min \left\{\sum_{i \in N} P_{j}\left|B T_{j}-B T_{j}^{*}\right|+\sum_{m \in M} P_{m}\left|y_{m}\right|\right\}
\end{gathered}
$$$$
B T_{i r_{i} j m} \geq 0
$$

$\sum_{i=1}^{I} \sum_{r_{i}=1}^{R_{i}}\left(P_{i r_{i} j m, k} * D_{r_{i}}\right) \leq A C_{m} *\left(T_{k+1}-T_{k}\right)$

$0 \leq \omega_{i} \leq 1$

$\sum_{i=1}^{I} \omega_{i}=1$

$H=\sum_{k=1}^{p-1}\left(T_{k+1}-T_{k}\right)+T_{1}$,

$k=1,2, \cdots, p-1$, and integer

In the model, formula (3) stands for the makespan of workpiece; formula(4)stands for weighing the total tardiness of every workpiece, where ${ }^{\omega_{i}}$ stands for tardiness penalty coefficient of $\operatorname{task}{ }^{i} ;{ }^{P_{j}}$ in formula(5)stands for penalty factor arising from change of start machining time of procedure ${ }^{j}, P_{m}$ is the machine penalty factor arising from procedure change in initial scheduling. For simplification purpose, take its value as 1 in below examples, which stands for the indicator of initial scheduling deviation in the system; constraint (6) stands for start time of workpiece in any procedure which if nonegative; constraint (7) stands for any procedure ${ }^{j}$ of the same workpiece can only start machining after procedure ${ }^{j-1}$ which is completed; $i^{*}$ in constraint (8) is any workpiece but workpiece ${ }^{i}, m^{*}$ is any 
equipment but equipment $m$, so this formula stands for start machining time of any workpiece ${ }^{i}$ in No. $k$ rescheduling interval on equipment $m$ in procedure ${ }^{j}$ under rout . There are two cases depending on whether it is the first procedure of the workpiece or not. The beginning time of $k$ rescheduling interval depends on the larger between rescheduling time ${ }^{T_{k}}$ and machining termination time of machining equipment if it is. If not, the beginning time of $k$ rescheduling interval depends on the larger between terminal time of the last procedure and machining termination time of machining equipment needed by the procedure of the workpiece; constraint (9) stands for terminal time of any procedure being the sum of the start time and machining time; constraint (10) stands for that workpiece machining in any rescheduling interval needs to meet the constraint of equipment capacity; constrain (11) stands for the weight of each workpiece tardiness weighing is no less than 0 and no more than 1 ; constraint (12) stands for the sum of each weight is 1 ; constraint (13) is that of the length of scheduling interval, the $\mathrm{P}$ in the formula stands for total time phase of scheduling interval division.

\section{Algorithm design}

With the number of tasks, devices, virtual cellulars and planning periods, the solving process will be very complicated. Therefore, the multi-objective optimization algorithm based on genetic algorithm is used to solve the model.

\subsection{Encoding and Decoding}

Take five types of machine as an example to illustrate.In Fig.1, the integers 1, 2, 3 in the task row represent the unique number of the workpiece. The position in the code in the selection line of equipment indicates the type of machine which is to be processed for the corresponding process. The first one of the rows indicates that the first step of the workpiece 1 is processed on the machine 3 . The first step in the row 1 of the workpiece 1 is processed in virtual cellular 2.

\begin{tabular}{|c|l|l|l|l|l|l|l|l|l|}
\hline Task order & \begin{tabular}{|l|l|l|l|l|l|l|l|l|}
\hline 1 & 3 & 1 & 2 & 2 & 3 & 2 & 1 & 3 \\
\hline $\begin{array}{c}\text { Machine } \\
\text { number }\end{array}$
\end{tabular} \\
\begin{tabular}{|l|l|l|l|l|l|l|l|l|}
\hline $\begin{array}{c}\text { Cell } \\
\text { number }\end{array}$ \\
\hline
\end{tabular} & 4 & 2 & 1 & 5 & 2 & 1 & 1 & 3 \\
\hline
\end{tabular}

Fig. 1 encoding and decoding

\subsection{Operations of Cross and Mutation}

In order to enable the updated population to satisfy the model, a cross-operation based on process coding (POX) is adopted. First, select two chromosomes that need to be crossed. Second, the number of genes in the second chromosome is recorded and inserted into the corresponding position of the first chromosome. Finally, the remaining genes in the first chromosome are sequentially placed into the remaining gene positions. Thus, the cross operation is implemented, and the graph is a POX cross raised in the encoding. In order to maintain the diversity of groups, using the insertion mutation, that is, randomly selected a gene or gene fragment, inserted into a different random location.

\section{Case Analysis}

Take X shipbuilding's actual machining workshop production tasks for example. The scheduling cycle is 100 hours. Table 1shows the relevant information of equipment.Table 2 shows the process information of the original processing task. Table 3 shows the processing information of new tasks, and the initial schedule Gantt chart is shown in fig.2.

When the new task $\mathrm{P}_{6}$ arrives, indexes of the two trigger were calculated, $\bar{U}_{\mathrm{m}}=30.21 \%, \bar{T}>0$. According to the above method, it calculates the indexes of two trigger respectively in the time of 24 hours and 100 hours. When the time is 24 hours, we can get the indexes $\bar{U}_{\mathrm{m}}<30 \%, \bar{T}>0$, and so rescheduling is triggered. When the time is 100 hours, we can get the indexes $\bar{U}_{\mathrm{m}}<30 \%, \bar{T}>0$, and so rescheduling is triggered. The reschedule Gantt chart which considered new tasks is shown as fig.4. 
Table.1 Basic information of the machine and equipment

\begin{tabular}{cccc}
\hline Operation Names & Machine ID & Machine Types & Machine Count \\
\hline \multirow{3}{*}{ Cutting } & $\mathrm{M}_{1}$ & C534J & 1 \\
& $\mathrm{M}_{2}$ & CAK6180B & 1 \\
& $\mathrm{M}_{3}$ & DL160 & 1 \\
& $\mathrm{M}_{4}$ & T612A & 1 \\
& $\mathrm{M}_{5}$ & TPX6113 & 1 \\
\multirow{5}{*}{ Milling } & $\mathrm{M}_{6}$ & T6216C & 1 \\
& $\mathrm{M}_{7}$ & T6920D/L100 & 1 \\
& $\mathrm{M}_{8}$ & T6920F/120 & 1 \\
\multirow{3}{*}{ Grindig } & $\mathrm{M}_{9}$ & M7150 & 1 \\
\hline
\end{tabular}

Table.2 Original orders information of processing

\begin{tabular}{|c|c|c|c|c|c|}
\hline Tasks & Workpiece & Optional machine & Number & Processing time/hours & Dating time/hours \\
\hline \multirow{4}{*}{$\mathrm{P}_{1}$} & $\mathrm{P}_{1-1}$ & $\mathrm{M}_{1} / \mathrm{M}_{2}$ & \multirow{4}{*}{4} & $1.8 / 1.6$ & \multirow{4}{*}{140} \\
\hline & $\mathrm{P}_{1-2}$ & $\mathrm{M}_{3}$ & & 3.8 & \\
\hline & $P_{1-3}$ & $\mathrm{M}_{7} / \mathrm{M}_{8}$ & & $4.2 / 4.175$ & \\
\hline & $P_{1-4}$ & $\mathrm{M}_{9}$ & & 2.4 & \\
\hline \multirow{5}{*}{$\mathrm{P}_{2}$} & $\mathrm{P}_{2-1}$ & $\mathrm{M}_{1} / \mathrm{M}_{2}$ & & $3 / 3.2$ & \multirow{5}{*}{140} \\
\hline & $\mathrm{P}_{2}-2$ & $\mathrm{M}_{3}$ & & 3 & \\
\hline & $\mathrm{P}_{2-3}$ & $\mathrm{M}_{7} / \mathrm{M}_{8}$ & 8 & $3 / 3.2$ & \\
\hline & $\mathrm{P}_{2}-4$ & $\mathrm{M}_{9}$ & & 3.6 & \\
\hline & $\mathrm{P}_{2-5}$ & $\mathrm{M}_{10}$ & & 2.4 & \\
\hline \multirow{4}{*}{$\mathrm{P}_{3}$} & $\mathrm{P}_{3-1}$ & $\mathrm{M}_{1} / \mathrm{M}_{2}$ & \multirow{5}{*}{5} & $1.52 / 1.5$ & \multirow{5}{*}{140} \\
\hline & $\mathrm{P}_{3}-2$ & $\mathrm{M}_{3}$ & & 2.1 & \\
\hline & $\mathrm{P}_{3}-3$ & $\mathrm{M}_{7} / \mathrm{M}_{8}$ & & $6.72 / 5.8$ & \\
\hline & $\mathrm{P}_{3}-4$ & $\mathrm{M}_{10}$ & & 2.52 & \\
\hline \multirow{5}{*}{$\mathrm{P}_{4}$} & $\mathrm{P}_{4-1}$ & $\mathrm{M}_{1} / \mathrm{M}_{2}$ & & $3 / 3.2$ & \\
\hline & $\mathrm{P}_{4-2}$ & $\mathrm{M}_{4} / \mathrm{M}_{5} / \mathrm{M}_{6}$ & & $3.6 / 3.2 / 3.4$ & \multirow{4}{*}{140} \\
\hline & $\mathrm{P}_{4-3}$ & $\mathrm{M}_{3}$ & 5 & 1.8 & \\
\hline & $\mathrm{P}_{4-4}$ & $\mathbf{M}_{10}$ & & 3.36 & \\
\hline & $P_{4-5}$ & $\mathrm{M}_{7} / \mathrm{M}_{8}$ & & $5.4 / 5.2$ & \\
\hline \multirow{3}{*}{$\mathrm{P}_{5}$} & $\mathrm{P}_{5-1}$ & $\mathrm{M}_{1} / \mathrm{M}_{2}$ & & $4.5 / 4.8$ & \multirow{3}{*}{140} \\
\hline & $\mathrm{P}_{5-2}$ & $\mathbf{M}_{10}$ & 4 & 4.5 & \\
\hline & $\mathrm{P}_{5-3}$ & $\mathrm{M}_{3}$ & & 2.25 & \\
\hline
\end{tabular}

Table. 3 New orders information of processing

\begin{tabular}{ccccccc}
\hline Tasks & workpiece & Optional machine & Number & $\begin{array}{c}\text { Processing } \\
\text { time/hours }\end{array}$ & $\begin{array}{c}\text { Arriving } \\
\text { time/hours }\end{array}$ & $\begin{array}{c}\text { Dating } \\
\text { time/hours }\end{array}$ \\
\hline \multirow{4}{*}{$\mathrm{P}_{6}$} & $\mathrm{P}_{6}-1$ & $\mathrm{M}_{1} / \mathrm{M}_{2}$ & & $1.2 / 1.6$ & & \\
& $\mathrm{P}_{6}-2$ & $\mathrm{M}_{4} / \mathrm{M}_{5} / \mathrm{M}_{6}$ & & $1.5 / 1.3 / 1.6$ & & \\
& $\mathrm{P}_{6}-3$ & $\mathrm{M}_{1} / \mathrm{M}_{2}$ & 2 & $1.5 / 1.8$ & & \\
& $\mathrm{P}_{6}-4$ & $\mathrm{M}_{7} / \mathrm{M}_{8}$ & & $2.2 / 2.4$ & & \\
& $\mathrm{P}_{6}-5$ & $\mathrm{M}_{10}$ & & 1.8 & & \\
& $\mathrm{P}_{7}-1$ & $\mathrm{M}_{1} / \mathrm{M}_{2}$ & & $2.4 / 2.2$ & & \\
$\mathrm{P}_{7}$ & $\mathrm{P}_{7}-2$ & $\mathrm{M}_{4} / \mathrm{M}_{5} / \mathrm{M}_{6}$ & \multirow{2}{*}{3} & $2.4 / 2.5 / 2.7$ & & \\
& $\mathrm{P}_{7-3}$ & $\mathrm{M}_{10}$ & 3 & 7.2 & & \\
& $\mathrm{P}_{7}-4$ & $\mathrm{M}_{9}$ & & 0.6 & & \\
\hline
\end{tabular}




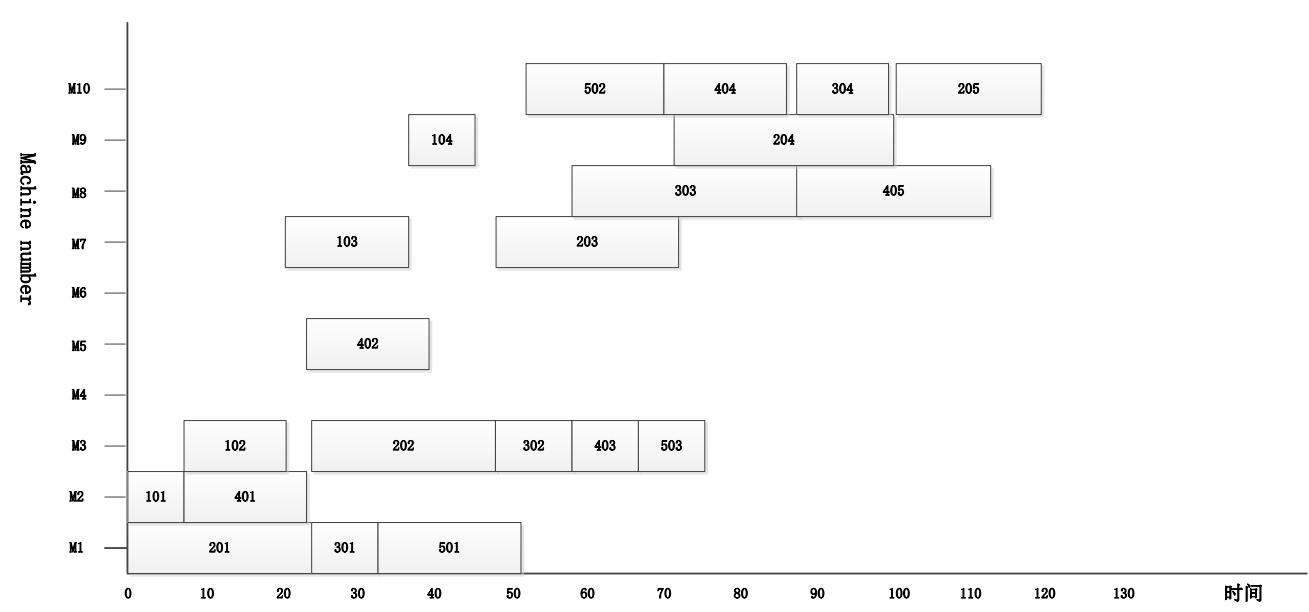

Fig.2 the original schedule Gantt chart

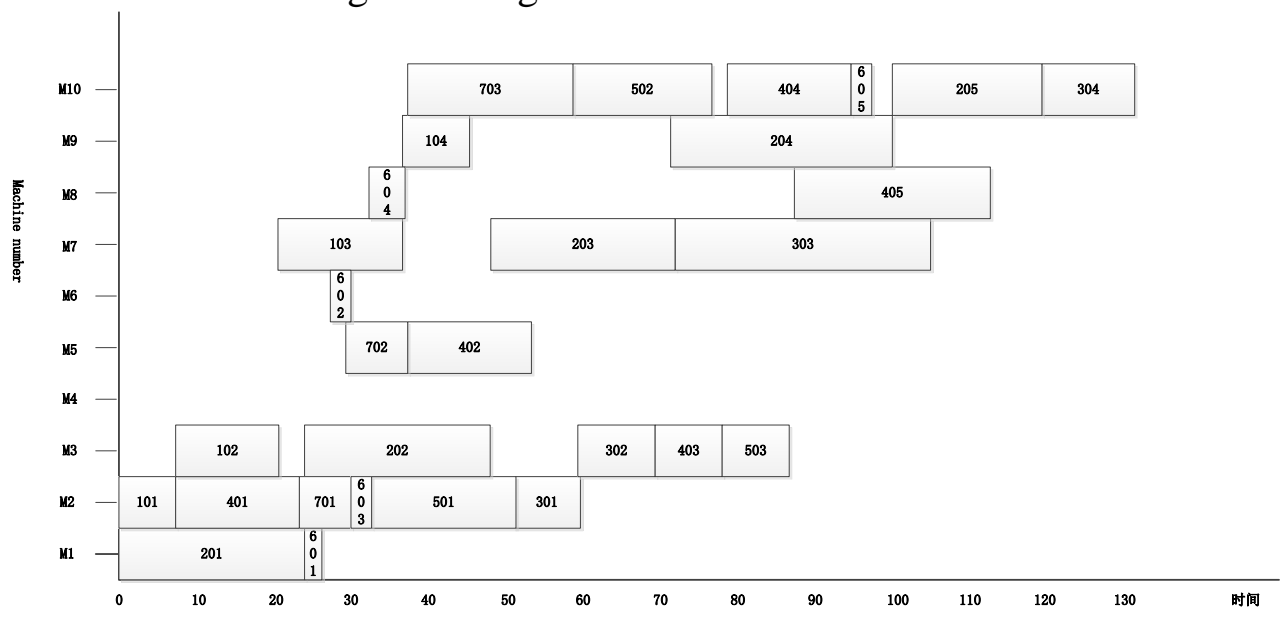

Fig.3The rescheduling Gantt chart considering new orders arriving

By adopting the proposed hybrid driven under the two trigger for scheduling decision making, the final completion time was 132.6 hours, a total of rescheduling twice. And under the hybrid driven, we should use the same method of multi-objective genetic algorithm to reschedule for 3 times and the final completion time was 132.8 hours.

\section{Conclusion}

In this paper, the dynamic scheduling interval is divided into several static by combining rolling window technology. Multiple targets rescheduling of model is proposed to optimize scheduling of each interval. By analyzing the above example, it shows that the decision making method proposed in this paper can effectively reduce the number of unnecessary rescheduling on the basis of not increasing makespan, so as to lowering computing cost and resetting cost due to rescheduling, increase system stability. At the same time, it also improves quality of rescheduling and increases the average utilization of machine.

\section{References}

[1]. Han W M,Gao L L,Weng H B,et al.Research on Virtual Cell Scheduling of Pipe Processing in Ship Building Based on Theory of Blocking Flow[J].Ship building of China, 2016,57(3): 164-175.

[2]. Driss I, Mouss K. A new genetic algorithm for flexible job-shop scheduling problems [J]. Journal of Mechanical Science and Technology, 2015, 29(3):1273-1281. 
[3]. Wang X Q, Tang J F, Yung K L. A scatter search approach with dispatching rules for a joint decision of cell formation and parts scheduling in batches[J]. International Journal of Production Research, 2010, 48(12):3513-3534.

[4]. Adibi M A, Zandieh M, Amiri M. Multi-objective scheduling of dynamic job shop using variable neighborhood search[J]. Expert Systems with Applications, 2010, 37(1):282-287.

[5]. Zhang J,Qin W,Song D L.Rescheduling Algorithm Based on Rolling Horizon Procedure for a Dynamic Hybrid Flow Shop with Uncertain Processing Time[J].Journal of Mechanical Engineering, 2015,51(11):99-108.

[6]. Yin Y, Cheng T C E, Wang D J. Rescheduling on identical parallel machines with machine disruptions to minimize total completion time[J]. European Journal of Operational Research, 2016, 252(3):737-749.

[7]. Qiao F, Wu Q, Li L. A fuzzy Petri net-based reasoning method for rescheduling[J].Transactions of the Institute of Measurement \& Control, 2009, 31(5):435-455.

[8]. Liu M Z, Zhang X, Zhang,et al. Rescheduling decision method of manufacturing shop based on profit-loss cloud model[J].Control and Decision,2014,29(8):1458-1464.

[9]. Gao K Z, Suganthan P N, Pan Q K, et al. Artificial Bee Colony Algorithm for Scheduling and Rescheduling Fuzzy Flexible Job Shop Problem with New Job Insertion[J]. Knowledge- Based Systems, 2016, 109:1-16.

[10]. Al-Hinai N, Elmekkawy T Y. Robust and stable flexible job shop scheduling with random machine breakdowns using a hybrid genetic algorithm[J]. International Journal of Production Economics, 2011, 132(2):279-291.

[11]. Jin L, Zhang C, Shao X, et al. A study on the impact of periodic and event-driven rescheduling on a manufacturing system: An integrated process planning and scheduling case[J]. Proceedings of the Institution of Mechanical Engineers Part B Journal of Engineering Manufacture, 2016:1-15. 